\title{
Social Determinants and Oral Health: An Update
}

\author{
Marisol Tellez • Avi Zini • Saskia Estupiñan-Day
}

Published online: 13 June 2014

(C) Springer International Publishing AG 2014

\begin{abstract}
This review presents the most recent studies and evidence on the social determinants of oral health. Oral disease is associated with an array of structural determinants (income, goods, and services). It is also associated with daily living conditions, and social gradients have been reported for dental caries, periodontal disease, oral cancer, and tooth loss. Current understanding of these interconnections have prompted the development of a new approach for oral health promotion, which recognizes that the behaviors accounting for the most important noncommunicable diseases contribute to oral diseases as well. Oral diseases and issues such as poor access to dental care and low oral health literacy levels are social, political, behavioral, and medical in nature. These conditions will only be controlled by the promotion of initiatives that prioritize the improvement in the social determinants of health as a backbone structure for the development of healthy enabling environments.
\end{abstract}

Keywords Social determinants of health $\cdot$ Socio-economic status $\cdot$ Oral health disparities $\cdot$ Dental caries $\cdot$ Periodontal disease $\cdot$ Oral cancer $\cdot$ Oral health literacy $\cdot$ Access to dental care

M. Tellez $(\bowtie)$

Pediatric Dentistry and Community Oral Health Sciences, Maurice H Kornberg School of Dentistry, 3223 N Broad Street, Room 307, Philadelphia, PA 19140, USA

e-mail: Marisol@dental.temple.edu

\section{A. Zini}

Department of Community Dentistry, Faculty of Dental Medicine, Hadassah - Hebrew University Medical Center, Jerusalem, Israel e-mail: AviZ@hadassah.org.il

\section{S. Estupiñan-Day}

Regional Oral Health Advisor, Pan American Health Organization, Washington, DC, USA

e-mail: estupins@paho.org

\section{Introduction}

In a time of health system transformation, greater attention is now being paid to access, utilization, and quality of health care and its effects on population health [1]. Similarly, research and case studies highlighting the importance of poverty, the physical environment, and educational attainment among others on health outcomes provide a better understanding of the complex social and structural determinants of health [1]. The landmark 2008 report of the World Health Organization's (WHO) Commission on Social Determinants of Health [2] and the "The Marmot report" for the UK in 2010 [3] clearly showed evidence for a social gradient in health, which is closely related to the social and economic factors that determine the conditions of daily life. The place in the social hierarchy that individuals and groups occupy, in addition to the environment, then determines exposure to healthenhancing or health-damaging conditions in daily life (e.g., where people are born, grow, live, work, and age) [4••].

Impacts of social determinants of health (SDH) can be accumulated during a lifetime, alter health trajectories across the life course, and be transferred across generations [5]. Despite acknowledgement that there are multiple sectors that contribute to the SDH, many actions to address these factors have traditionally been generated from the health systems only, excluding the education, economic, and environmental sectors among others. Multisector approaches to reducing the SDH impact [6 $6^{\circ}$ are much needed and dentistry is not an exception.

The term "social determinants" is relevant to communicable and noncommunicable diseases (NCDs) alike. Oral diseases share the same determinants and risk factors as the main NCDs comprising heart disease, cancer, chronic obstructive pulmonary disease, diabetes, dementia, and stroke. We know many oral diseases is associated with socioeconomic status, which links to family income, educational attainment, 
employment status, housing, physical health, and mental health. Children and young people in poorer areas have worse general and oral health than those in the more affluent neighborhoods [7]. Current understanding of these associations have prompted the development of a new approach for oral health promotion, which recognizes that the behaviors accounting for the most important NCDs contribute to oral diseases as well. The "Common Risk Factor Approach" (CRFA) is directed to reducing risk factors common to a number of NCDs, [8,9••] and addresses the SDH from an integrated and comprehensive health care approach. This review presents the most recent studies and evidence on the social determinants of oral health.

\section{Health Disparities and Oral Health}

There are significant disparities in oral health worldwide. Oral health disparities are most commonly reported for caries, chronic periodontitis, and cancers of the head and neck, as well as for receipt of preventive dental visits, sealants, tooth loss, and quality of life $[10,11]$. Moreover, the increase in dental caries and periodontal disease, particularly among the elderly and children, indicate that the causes of those diseases are not being controlled [11]. Hence, there is an urgent need for oral health policy to emphasize translational research and reinforce public health approaches to tackling the 'causes of the causes' addressing common risk factors [8, 9••]. Several definitions of health disparities exist, but they all have a profound connection with the SDH [12], as these health differentials are closely linked with social, economic, and/or environmental disadvantage, and, in some countries, to race, ethnicity and education $[4 \bullet \bullet, 13]$. Furthermore, the selfperceived social standing has come up as a significant determinant of health disparities in more recent analyses conducted with data from Australian adults [14].

Taking into consideration the constellation of proximal and distal determinants of oral health, it has been suggested that population level approaches may be superior to high-risk group identification and targeting, though combinations of both strategies can be most valuable $[15,16]$. Actions and efforts addressing "upstream" causes are critical from ethical, human rights, and social justice perspectives. They also confer equitable health promotion benefits in substantial population groups [17] and not only in high-risk groups. The former strategy has failed to reduce the oral disease burden gap between disadvantaged and more affluent groups of the population [15].

Policy and systems change is essential for reducing health disparities and creating communities of opportunity that support good health. Local policy work that is rooted in community partnerships and cross-sector collaborations is a key part of achieving this mission [18]. There is a fundamental need to integrate initiatives to improve oral health with more general interventions to support good physical and mental health. Primary care is the first point of contact with the health service and is the setting in which most general and oral healthcare is provided. Oral health teams have the largely unexploited potential to be important advocates, enablers, and mediators for oral and general health and for the reduction of disparities because the risk factors for oral and general health are the same [7]. Legislative, regulatory, and fiscal policies can be implemented to promote and maintain oral health through creating caring local environments especially in settings such as preschools so that positive and supportive early life experiences are fostered.

\section{Dental Caries}

Costa and colleagues [19] recently carried out a systematic review of the relationship between socioeconomic indicators and dental caries and found that, across 41 studies, education, income, occupation, and the Gini coefficient (measure of income or wealth inequality) were associated with higher rates of caries. Along these lines, Bernabé and Hobdell [20] analyzed data from 48 countries and found that, among affluent countries, income inequality may be a stronger determinant of childhood dental caries than is absolute income. In the United States, racial and ethnic disparities are prevalent for a variety of oral health indicators across various age groups, especially for untreated dental caries among children and adolescents. Data from the National Health Nutrition and Examination Survey, 2009-2010 showed that among children aged 3-5 and 6-9 years, untreated caries is significantly higher for those living at or below the federal poverty level compared with those living above the poverty threshold [10]. Early childhood caries continues to be a social, political, behavioral, and medical problem that can be controlled only through understanding the vigorous changes that are taking place in society, particularly those related to the environment such as neighborhood, family structure, nurturing of children, and socioeconomic status [21].

\section{Periodontal Diseases}

Periodontal diseases are highly prevalent, particularly amongst socially disadvantaged populations, impact on quality of life and are costly to treat. Populations generally at risk are characterized by inequity related to factors such as low income, less years of education, and black or mixed color ethnicity [22]. Moreover, these disadvantages might exist among all age groups. The prevalence of adverse periodontal conditions among adolescents has been associated with the color/race, type, and location of school, [23], while a cross 
sectional study found significant differences in adults' oral health between welfare state regimes [24]. A study among adults 18-64 years old reported that SDH such as social security and health insurance, low literacy levels, dental care utilization, and other behavioral aspects such as high tobacco consumption were major risk factors for periodontal and other oral diseases among groups with similar socioeconomic status [25]. Furthermore, a longitudinal study among a similar age group reported that level of education, income, and marital status were associated with periodontitis and tooth loss [26]. A distinct social aspect is religiosity. Religiosity has been described as a protective factor against periodontal diseases through extrinsic factors, by higher social support, and intrinsic factors, by higher spirituality pathways [27].

Clinical treatments and chair side preventive approaches alone will never adequately address health differentials related to periodontal diseases. Indeed, in many parts of the developing world, clinical care and chair side prevention are both unaffordable and inappropriate for the control of periodontal diseases. A recent review suggested that selling and dispensing such resources as toothbrushes and nicotine replacement therapies at cost price to patients can prevent periodontal diseases among socially disadvantaged groups [28].

A paradigm shift away from the individualized treatment approach to a population public health model is needed to promote periodontal health and, in particular, to address social inequalities in periodontal status. Public health measures need to focus on the underlying determinants of periodontal diseases. Poor hygiene, tobacco use, psychosocial factors, and related systemic diseases are the main risk factors for periodontal diseases. Public health interventions need to tackle these factors but also must recognize and act upon the distal underlying influences that determine and pattern these identified risks globally $[28,29]$. Finally, recognition also needs to be placed on the interlinking and common risk factors shared by periodontal diseases and other chronic conditions [29].

\section{Oral Cancer}

Head and neck cancers are a cause of morbidity and mortality throughout the United States [30, 31], and worldwide [32]. For low income African-American men, head and neck cancers are one of the leading causes of excess cancer deaths; although all racial/ethnic groups suffer from these cancers, African-American men disproportionately suffer and die from them $[30,31]$.

Low income communities have increased risks for many adverse health conditions because of environmental factors and existing SDH. These determinants often lead to unhealthy life circumstances and ineffective coping behaviors. These communities most often live in high-risk areas with various difficulties as the provision of health care is limited [33].
Risk factors for head and neck cancer, such as tobacco and alcohol use, are likely to be increased in low income communities, due to uncontrolled social determinants [33]. A study that aimed to examine the role of tobacco and alcohol in the incidence of oral cancer highlighted the importance of an awareness campaigns among smokers and heavy drinkers as cancer is more frequent among these underserved populations [34].

Early detection enhances effective treatment options, but such services are limited among African-Americans, particularly men in underserved areas. Not addressing oral cancer because of these social conditions reflects unjust and unethical behavior by health care professionals [33].

The inequality in oral cancer by social determinants is expressed not only by incidence rates but also by long-term quality of life after oncologic surgery [35]. In a recent review, an increased risk for oral cancer was seen among disadvantaged socioprofessional groups, and different levels of education [36]. Oral cancer is a preventable and treatable disease, when it is diagnosed in time. Screening should be performed only when the cost-effectiveness ratio is justified and has been established [37].

\section{Dental Access}

While some data show improvements in the U.S in oral health status overall, underserved and vulnerable populations continue to suffer in their access to needed services. The proportion of the population reporting being unable to get or delaying needed dental care is higher than the percentage of the population unable to get or delaying needed medical care or prescription drugs [38]. Furthermore, Fisher-Owens et al. [39] used 2007 National Survey of Children's Health data and found that racial/ethnic disparities in children's oral health status and health care access were attributable largely to socioeconomic and health insurance factors, suggesting that reducing health disparities may be more efficacious if efforts are targeted to distal determinants. In another recent study by Iida and Rozier [40], the investigators utilized U.S. representative data to show that mothers' (low) perceived social capital (the collective value of all social networks) was associated with both less frequent preventive dental visits and more unmet dental needs in their children.

\section{Oral Health Literacy}

Health literacy is critical because it can affect health care use, health outcomes, and overall dental care costs. The misconception that oral health is less important than general health exists among America's citizens even though it has been 13 years since the Surgeon General's report affirmed oral 
health as an essential component of general health. Research has shown that poor oral health literacy affects oral health, can negatively influence the quality of life, and has a significant financial impact on society. National initiatives to increase the oral health literacy levels of American citizens include training health care professionals about effective communication skills and disseminating oral health information to groups outside of dentistry [41]. Poor oral health literacy contributes to poor access because individuals may not understand the importance of oral diseases and oral health care or their options for accessing such care [42]. As the education system is where most people develop basic literacy skills and health related knowledge, it is fundamental that multisectorial approaches are developed to both improve oral health literacy and reduce disparities.

\section{Oral Health and General Health}

The connections between oral health and overall health and its implications for poor oral health are strong. However, dentistry remains substantially separated from the rest of health care, and oral health care is often overlooked in policy discussions about the nation's health care system. Some efforts have been made to introduce basic oral health care into primary health care, but we still need to capitalize on these efforts so improved and sustainable community care models addressing oral diseases and NCD's become well established. Particularly where resources are scarce, dentists are uniquely positioned to combine their force with primary care providers in prevention strategies $[8,9 \cdot \bullet]$.

\section{Conclusion}

Most of the factors influencing health; such as experiences in the early years, education, working life, income, and environmental conditions, lie outside the immediate reach of the health system. Health professionals, however, have an important role to play in tackling health disparities amongst their own patients and more widely in the community [7] and promoting health equity [43]. Current understanding of the social determinants indicates that significant improvements in health could be achieved if medical, dental, and public health professionals address these broader influences on health outcomes while maintaining excellence in traditional disease control approaches [1]. Oral diseases like dental caries, periodontal disease and oral cancer, issues such as poor access to dental care, and low oral health literacy levels are social, political, behavioral, and medical in nature. These conditions will only be controlled by the promotion of initiatives that prioritize the improvement in the SDH as a backbone structure for the development of healthy enabling environments. New models for oral health care are needed that allocate much more resources to population based promotion directed at the social determinants of risk factors common to NCDs [44]. Unless these determinants are addressed, improving access to dental care, will only alleviate but never substantially reduce, the burden of dental diseases.

\section{Compliance with Ethics Guidelines}

Conflict of Interest Dr. Marisol Tellez, Dr. Avraham Zini, and Dr. Saskia Estupiñan-Day each declare no potential conflicts of interest.

Human and Animal Rights and Informed Consent This article does not contain any studies with human or animal subjects performed by any of the authors.

\section{References}

Papers of particular interest, published recently, have been highlighted as:

- Of importance

•- Of major importance

1. Dean HD, Williams KM, Fenton KA. From theory to action: applying social determinants of health to public health practice. Public Health Rep. 2013;128 Suppl 3:1-4.

2. Commission on Social Determinants of Health. CSDH final report: closing the gap in a generation: health equity through action on the social determinants of health. Geneva: World Health Organization; 2008.

3. Marmot M, Atkinson T, Bell J, et al. Fair society, healthy lives: the Marmot Review. Strategic review of health inequalities in England post-2010 (The Marmot Review: UK). 2010. http://www. instituteofhealthequity.org/projects/fair-society-healthy-lives-themarmot-review. Accessed 4 Mar 2014.

4.• Lee JY, Divaris K. The ethical imperative of addressing oral health disparities: a unifying framework. J Dent Res. 2013;4:1-7. This manuscript provides a conceptual framework outlining the important role of the social determinants of oral health and illustrate pathways that may operate across the continuum of these determinants.

5. Sadana R, Blas E. What can public health programs do to improve health equity? Public Health Rep. 2013;128 Suppl 3:12-20.

6. Russell E, Johnson B, Larsen H, et al. Health systems in context: a systematic review of the integration of the social determinants of health within health systems frameworks. Rev Panam Salud Publica. 2013;34(6):461-7. This manuscripts reviews health systems frameworks in relation to social determinants of health and their impact in health outcomes.

7. Williams DM, Sheiham A, Watt RG. Oral health professionals and social determinants. Br Dent J. 2013;214(9):427.

8. FDI. Oral health and the United Nations Political Declaration on NCDs. A guide to advocacy http://www.fdiworldental.org/media/ 9465/oral health and_un political_dec_on_ncds.pdf. Accessed 4 Mar 2014.

9.• FDI. Policy Statement Non-Communicable Diseases Adopted by the FDI General Assembly: 31 August 2012 - Hong Kong. http:// www.fdiworldental.org/media/11291/Non-communicable $\%$ 20diseases-2012.pdf. Accessed 4 Mar 2014. This policy statement reflects the current views of the International Dental Federation 
regarding the links between oral diseases and noncommunicable diseases.

10. Dye BA, Li X, Thorton-Evans G. Oral health disparities as determined by selected healthy people 2020 oral health objectives for the United States, 2009-2010. NCHS Data Brief. 2012;104:1-8.

11. Thomson WM. Social inequality in oral health. Community Dent Oral Epidemiol. 2012;40 Suppl 2:28-32.

12. HHS 2010. Healthy People 2020: oral health objectives. http:// healthypeople.gov/2020/topicsobjectives2020/objectiveslist.aspx? topicid=32. Accessed 6 Mar 2014.

13. Do LG. Distribution of caries in children: variations between and within populations. J Dent Res. 2012;91:536-43.

14. Armfield JM, Mejía GC, Jamieson LM. Socioeconomic and psychosocial correlates of oral health. Int Dent J. 2013;63:202-9.

15. Watt RG, Sheiham A. Integrating the common risk factor approach into a social determinants framework. Community Dent Oral Epidemiol. 2012;40:289-96.

16. Sheiham A, Alexander D, Cohen L, et al. Global oral health inequalities: task group-implementation and delivery of oral health strategies. Adv Dent Res. 2011;23:259-67.

17. Rose G. Sick individuals and sick populations. Int J Epidemiol. 1985;14:32-8.

18. Schaff K, Desautels A, Flournoy R, et al. Addressing the social determinants of health through the Alameda County, California Place Matters Policy Initiative. Public Health Rep. 2013;128 Suppl 3:48-53.

19. Costa SM, Martins CC, Bonfim M d L, et al. A systematic review of socioeconomic indicators and dental caries in adults. Int J Environ Res Public Health. 2012;9:3540-74.

20. Bernabé E, Hobdell MH. Is income inequality related to childhood dental caries in rich countries? J Am Dent Assoc. 2010;141:143-9.

21. Ismail AI, Sohn W. A systematic review of clinical diagnostic criteria of early childhood caries. J Public Health Dent. 1999;59: 171-9.

22. Bonfim MD, Mattos FF, Ferreira EF, et al. Social determinants of health and periodontal disease in Brazilian adults: a cross- sectional study. BMC Oral Health. 2013;13(1):22.

23. Ribeiro Jordão LM, Vasconcelos DN, Moreira RDS, et al. Individual and contextual determinants of periodontal health in 12-year-old schoolchildren in a Brazilian Capital City. Int J Dent. 2012;325475. 7 pages.

24. Guarnizo-Herreño CC, Tsakos G, Sheiham A, Watt RG. Oral health and welfare state regimes: a cross-national analysis of European countries. Eur J Oral Sci. 2013;121:169-75.

25. Singh A, Purohit BM, Masih N, et al. Risk factors for oral diseases among workers with and without dental insurance in a national social security scheme in India. Int Dent J. 2013. doi:10.1111/idj. 12067.

26. Buchwald S, Kocher T, Biffar R, et al. Tooth loss and periodontitis by socio-economic status and inflammation in a longitudinal population-based study. J Clin Periodontol. 2013;40:203-11.

27. Zini A, Sgan-Cohen HD, Marcenes W. Is religiosity related to periodontal health among the adult Jewish population in Jerusalem? J Periodontal Res. 2012;47(4):418-25.
28. Watt RG, Petersen PE. Periodontal health through public health the case for oral health promotion. Periodontology 2000. 2012; 60: 147-55.

29. Petersen PE, Baehni PC. Periodontal health and global public health. Periodontology 2000. 2012; 60:7-14.

30. Kingsley K, O'Malley S, Ditmyer M, et al. Analysis of oral cancer epidemiology in the US reveals state-specific trends: implications for oral cancer prevention. BMC Public Health. 2008;8:87. doi:10. 1186/1471-2458-8-87.

31. Silverman S. Demographics and occurrence of oral and pharyngeal cancers. The outcomes, the trends, the challenge. JADA. 2001;132: $7 \mathrm{~s}-11 \mathrm{~s}$.

32. Warnakulasuriya S. Global epidemiology of oral and oropharyngeal cancer. Oral Oncol. 2009;45:309-16.

33. Moore CE, Warren R, Maclin Jr SD. Head and neck cancer disparity in underserved communities: probable causes and the ethics involved. J Health Care Poor Underserved. 2012;23(4 Suppl):88 103. doi:10.1353/hpu.2012.0165.

34. Radoï L, Paget-Bailly S, Cyr D, et al. Tobacco smoking, alcohol drinking and risk of oral cavity cancer by subsite: results of a French population-based case-control study, the ICARE study. Eur J Cancer Prev. 2013;22(3):268-76.

35. Pierre CS, Dassonville O, Chamorey E, et al. Long-term quality of life and its predictive factors after oncologic surgery and microvascular reconstruction in patients with oral or oropharyngeal cancer. Eur Arch Otorhinolaryngol. 2013;16. doi:10.1007/s00405-0132592-z.

36. Radoï L, Luce D. A review of risk factors for oral cavity cancer: the importance of a standardized case definition. Community Dent Oral Epidemiol. 2013;41:97-109.

37. Rethman MP, Carpenter W, Cohen EE, et al. Evidence-based clinical recommendations regarding screening for oral squamous cell carcinomas. JADA. 2010;141(5):509-20.

38. Chevarley FM. Percentage of persons unable to get or delayed in getting needed medical care, dental care, or prescription medicines: United States 2007. Statistical brief \#282. Rockville: Agency for Healthcare Research and Quality; 2010.

39. Fisher-Owens SA, Isong IA, Soobader MJ, et al. An examination of racial/ethnic disparities in children's oral health in the United States. J Public Health Dent. 2013;73:166-74.

40. Iida H, Rozier RG. Mother-perceived social capital and children's oral health and use of dental care in the United States. Am J Public Health. 2013;103:480-7.

41. Bress LE. Improving oral health literacy - the new standard in dental hygiene practice. J Dent Hyg. 2013;87(6):322-9.

42. IOM (Institute of Medicine). Advancing oral health in America. Washington, DC: The National Academies Press; 2011.

43. Dean HD, Fenton KA. Integrating a social determinants of health approach into public health practice: a five-year perspective of actions implemented by CDC's national center for HIV/AIDS, viral hepatitis, STD, and TB prevention. Public Health Rep. 2013;128 Suppl 3:5-11.

44. Sheiham A. Major changes in strategies are needed to promote oral health worldwide. J Public Health Dent. 2013;73(Issue 2):87-8. 